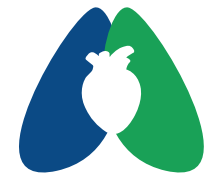

ASSOBRAFIR C I Ê N C I A

\title{
Fatores de risco associados com falha de extubação em uma unidade de terapia intensiva de trauma
}

\author{
Risk factors associated with extubation failure in a trauma \\ intensive care unit
}
Carolina Ocelli de Araujo Alves ${ }^{1}$ (i), Julia Alencar Renault ${ }^{1}$ (i) , Priscilla Ribeiro Soares ${ }^{1}$ (i), Ronaldo Angelo Dias da Silva² (1)
${ }^{1}$ Programa de Pós-graduação, Fundação Hospitalar do Estado de Minas Gerais (FHEMIG), Belo Horizonte, MG, Brasil
${ }^{2}$ Centro Universitário Estácio de Sá, Belo Horizonte, MG, Brasil

Como citar: Alves COA; Renault JA; Soares PR; Angelo R. Fatores de risco associados com falha de extubação em uma unidade de terapia intensiva de trauma. ASSOBRAFIR Ciênc. 2021;12:e43313. https://doi. org/10.47066/2177-9333.AC.2020.0020

\begin{abstract}
Resumo
Introdução: O desafio clínico atual é melhorar o desmame da ventilação mecânica invasiva (VMI) em pacientes criticamente doentes. Parâmetros comumente utilizados são descritos pela literatura e capazes de informar sobre a capacidade do doente em respirar espontaneamente. Objetivo: identificar os fatores de risco para predizer o desfecho da extubação em pacientes internados em uma unidade de terapia intensiva (UTI) de trauma. Método: Estudo de coorte prospectivo para analisar o desfecho reintubação em pacientes vítimas de trauma. Adultos intubados que necessitaram de VMI por pelo menos $24 \mathrm{~h}$ foram examinados e acompanhados até alta ou óbito. Para análise de dados foram empregados os testes de Mann Whitney, Qui-quadrado, teste exato de Fisher, e regressão logística binária múltipla. Resultados: Dos 262 pacientes que passaram por uma extubação planejada, 56 pacientes falharam $(21,4 \%)$. A reintubação esteve associada independentemente com idade avançada (50,23 $\pm 2,24$ anos), maior escore no APACHE II $(14,75 \pm 5,39)$, frequência respiratória (FR) elevada, intubação orotraqueal (IOT) por causas respiratórias $(\mathrm{OR}=4,4, \mathrm{IC} 95 \%=1,792-11,025, \mathrm{p}=0,04)$ e presença de doenças respiratórias $(O R=3,3, I C 95 \%=1,136-9,926, p=0,05)$. Além disso, pacientes que falharam a extubação tiveram maior tempo de internação $(19,27 \pm 9,90, p<0,001)$, maior taxa de mortalidade ( $R R=4,1$, IC95\%=2,87-6,19, p<0,001), necessidade de ventilação não invasiva (VNI) pós-extubação e traqueostomia. Conclusão: maior escore no APACHE II, FR elevada, IOT por causas respiratórias e presença de comorbidades respiratórias foram os fatores de risco independentes para reintubação. Insucesso na extubação planejada foi seguida de deterioração clínica, sugerindo um efeito específico da falha de extubação e reintubação nos desfechos dos pacientes.
\end{abstract}

Palavras-chave: Terapêutica; Manuseio das Vias Aéreas; Extubação; Respiração Artificial.

\begin{abstract}
Background: The current clinical challenge is to improve weaning from invasive mechanical ventilation (IMV) in critically ill patients. Risk factors associated with extubation failure are well described in the literature and able to inform about the patient's ability to breathe spontaneously. Aim: to identify risk factors to predict extubation's outcome in patients admitted in a trauma intensive care unit (ICU). Methods: observational prospective cohort study conducted to evaluate reintubation outcome in trauma patients. Adult patients who needed IMV for at least 24 hours were examined and followed up until discharge or death. Results: Of the 262 patients who underwent a planned extubation, 56 (21.4\%) failed. Reintubation was independently associated with advanced age (50.23 \pm 2.24 years), higher APACHE II score (14.75 \pm 5.39$)$, high respiratory rate, endotraqueal intubation for respiratory causes $(\mathrm{OR}=4.4,95 \% \mathrm{Cl}=1.792-11.025, \mathrm{p}=0.04)$ and the presence of underlying respiratory diseases $(\mathrm{OR}=3.3,95 \% \mathrm{Cl}=1.136-9.926, \mathrm{p}=0.05)$. In addition, patients who failed extubation had longer hospitalization time $(19.27 \pm 9.90, p<0.001)$, higher mortality rate $(R R=4.1$,
\end{abstract}

$95 \% \mathrm{Cl}=2.87-6.19, \mathrm{p}<0.001)$, need for NIV and evolution to tracheostomy. Conclusion: higher
Submissão em: Abril 12, 2021 Aceito em: Junho 07, 2021

Estudo realizado em: Hospital João XXIII, Belo Horizonte, Minas Gerais, Brasil. Aprovação ética: CAEE

15823819.0.0000.5119 da Fundação Hospitalar do Estado de Minas Gerais, n 4.314.870.

*Autor correspondente: Carolina Ocelli de Araújo Alves. Email: carolocelli08@gmail.com

Este é um artigo publicado em acesso aberto (Open Access) e distribuído sob a licença Creative Commons Attribution NonComercial ShareAlike License, que permite uso, distribuição e reprodução em qualquer meio, sem restrições desde que sem fins comerciais e que o trabalho original seja corretamente citado e de forma que não indique endosso ao trabalho feito. A 
APACHE II score, high respiratory rate, endotraqueal intubation due to respiratory causes and the presence of respiratory comorbidities were independent risk factors for reintubation. Failure in planned extubation was followed by clinical deterioration, suggesting a specific effect of extubation and reintubation failure on patient outcomes.

Keywords: Therapeutics; Airway Management; Airway Extubation; Respiration; Artificial.

\section{INTRODUÇÃO}

Intubação orotraqueal (IOT) e suporte ventilatório por meio de ventilação mecânica invasiva (VMI) são comumente utilizados como intervenção de amparo a vida para pacientes críticos ${ }^{1}$. A VMI prolongada tem sido associada a várias complicações ${ }^{2-5}$, portanto, o tempo de VMI deve ser o mais breve possível. Desmame é o processo de retirada gradual da VMI e envolve uma série de fatores associados à predição da capacidade do paciente em assumir ventilação espontânea ${ }^{6,7}$. A maioria dos pacientes apresentam um processo de desmame simples: quando a condição de base que o levou à necessidade de VMI está estável, sendo capaz de respirar espontaneamente, e termina quando acontece a retirada da via aérea artificial, evento conhecido como extubação ${ }^{7,8}$.

A falha no processo de extubação não pode ser presumida por variáveis isoladas já que existe heterogeneidade entre os pacientes, tornando-se necessário considerar as condições dos múltiplos sistemas envolvidos na respiração $0^{9,10}$. A falha de extubação ocorre quando há a necessidade de reintubação em menos de 48 horas após a primeira tentativa de extubação 2,11, entretanto, este critério é variável entre os estudos 2,12-14. Pacientes que falharam à extubação apresentam maiores taxas de morbimortalidade, por isso, identificar e monitorizar os fatores de risco associados à falha é importante para otimizar o desfecho clínico 1,2,15-17.

Parâmetros comumente utilizados com o objetivo de predizer sucesso na extubação são descritos pela literatura e capazes de informar sobre a capacidade em respirar espontaneamente. Dentre eles estão a capacidade de proteção de via aérea, presença do reflexo de tosse e pico de fluxo, hipersecretividade, Escala de Coma de Glasgow (ECG) > 8, oxigenação arterial e sucesso no teste de respiração espontânea (TRE) 2,7,18-20.

O desafio clínico atual é melhorar o desmame da VMI em pacientes críticos. Extubações realizadas precoce ou tardiamente estão associadas a risco aumentado de complicações, necessidade de traqueostomia, aumento da permanência em Unidade de Terapia Intensiva (UTI) e mortalidade ${ }^{21,22}$, portanto, decisões sobre o momento correto para realizá-las são cruciais durante o manejo de pacientes ${ }^{1,21}$.Em pacientes com trauma, essa avaliação pode ser ainda mais difícil visto que as indicações para uso da VMI são geralmente multifatoriais devido à presença de lesões que podem alterar sistemas biológicos, o estado cognitivo, a mecânica ventilatória, acarretando em lesões pulmonares ou de órgãos abdominais, e mobilidade reduzida ${ }^{23}$
Além disso, altos custos associados aos episódios de reintubação estão bem descritos na literatura e incluem maior tempo de internação, maiores chances de morbimortalidade, e complicações associadas à $\mathrm{VMI}^{4,24}$. Sendo assim, a decisão de interromper a VMI inclui equilibrar os benefícios de evitar a morbidade associada à VMI com o risco de morbidade decorrente das falhas de extubação. Por isso, identificar os fatores de risco associados com falha de extubação em pacientes vítimas de trauma pode acarretar em uma tomada de decisão mais assertiva do melhor momento para interrupção da VMI e/ou da antecipação do momento de extubação, podendo assim, diminuir todos os malefícios citados anteriormente.

Portanto, o objetivo deste estudo foi identificar os fatores de risco e suas associações, se existentes, para predizer o desfecho da extubação em pacientes internados em UTI de trauma.

\section{MÉTODOS}

Estudo observacional de coorte prospectivo conduzido durante onze meses (novembro de 2019 - outubro de 2020), com duração média de internação na UTI de $12,35 \pm 7,52$ dias. Este trabalho foi aprovado pelo CAEE 15823819.0.0000.5119 da Fundação Hospitalar do Estado de Minas Gerais, $n^{\circ}$ 4.314.870. O termo de consentimento livre e esclarecido (TCLE) foi dispensado.

A população do estudo foi constituída por todos os pacientes admitidos na UTI de trauma com idade superior a 18 anos, que necessitaram de VMI por mais de 24 horas, que passaram por extubação planejada e por teste de respiração espontânea (TRE) bem sucedido ${ }^{25}$. Todos os pacientes foram rastreados e acompanhados até a alta hospitalar ou óbito. Foram excluídos os pacientes com relatos de extubação acidental, auto-extubação ou extubação não planejada, extubação paliativa, mulheres grávidas, pacientes que foram reintubados após 48 horas, reintubação eletiva ou emergencial para realização de procedimentos cirúrgicos, que foram transferidos de hospital ou morreram antes da extubação.

Coleta de Dados. Foram coletados dados dos prontuários eletrônicos e dos passômetros (formulários de transferência de cuidado) da equipe da enfermagem e fisioterapia, dados demográficos (idade e sexo), Acute Physiology and Chronic Health Evaluation II - APACHE como indicador de gravidade, duração da VMI, tempo de internação na UTI, motivo da intubação, comorbidades, tipo de TRE, episódio de pneumonia associada à VM $(P A V)^{26,27}$ e/ou sepse antes e/ou após a extubação e reintubação. Do checklist de extubação, foram coletados 
os parâmetros clínicos: dados vitais - frequência cardíaca (FC), saturação de oxigênio (SpO2), frequência respiratória (FR) e pressão arterial média (PAM), ECG, capacidade de atender a comandos verbais simples, hipersecretividade (frequência de aspiração endotraqueal maior que uma vez a cada $2 \mathrm{~h}$ ), pico de fluxo da tosse - velocidade máxima alcançada pelo fluxo aéreo em uma tosse forçada (avaliado pelo aparelho Peak Flow Meter ou no próprio ventilador) ${ }^{23,28-30}$, avaliação subjetiva da tosse ${ }^{31}$ (escala numérica de 0 a 5, onde 0 corresponde a ausência de tosse ao comando; 1 movimento audível de ar por meio do tubo; 2 tosse audível, porém fraca; 3 tosse claramente audível; 4 tosse forte; e 5 tosses fortes sequenciais), pico de temperatura corporal e balanço hídrico nas 24 horas que precederam a extubação. Foram coletados também dados obtidos a partir da análise dos gases arteriais do sangue, $\mathrm{pH}$, hemoglobina, Proteína C-reativa (PCR) e leucócitos. Após a extubação, foram registradas as seguintes variáveis: sucesso ou falha de extubação, falha de extubação em até 48hs, motivo da reintubação, tempo de VMI após a reintubação, necessidade de Ventilação Mecânica Não-Invasiva (VNI), traqueostomia, e desfecho do paciente.

Análise Estatística. Para verificação da normalidade dos dados de variáveis quantitativas foi empregado o teste de Shapiro-Wilk. Não atendido o pressuposto de normalidade foi realizado o teste de Mann Whitney. A análise das variáveis categóricas foi realizada por meio do teste Qui-quadrado e quando o pressuposto de frequência esperada mínima foi menor que cinco, o teste exato de Fisher foi utilizado. Posteriormente, uma análise multivariada foi aplicada a todas as variáveis pré-extubação que atingiram um $a<0,20$ nas análises bivariadas. Tais variáveis foram incluídas como preditoras em um modelo de regressão logística binária múltipla, em que a variável resposta foi a reintubação. Além da estatística inferencial, foi realizada a análise descritiva para sumarizar os resultados das variáveis do estudo (média \pm desvio padrão, distribuição de frequência, valores percentuais e intervalo de confiança (IC95\%). O nível de significância adotado foi de $\mathrm{a}=0,05$. Para a análise estatística dos dados foi utilizado o software SPSS versão 20. Foram utilizados para análise estatística apenas prontuários com todos os dados preenchidos.

\section{RESULTADOS}

Setecentos e quarenta e quatro pacientes com necessidade de suporte ventilatório foram admitidos na UTI; sendo que 41 deles já estavam traqueostomizados à admissão e 703 receberam suporte ventilatório via tubo orotraqueal. 441 pacientes não atenderam aos critérios de inclusão ou foram excluídos. Dos 262 pacientes incluídos, 206 tiveram sucesso na extubação $(78,6 \%)$, enquanto 56 pacientes foram reintubados em menos de 48 horas $(21,4 \%)$ (Figura 1). A maioria dos pacientes incluídos eram homens $(72,1 \%)$ com média de 43,2 $\pm 17,75$ anos.

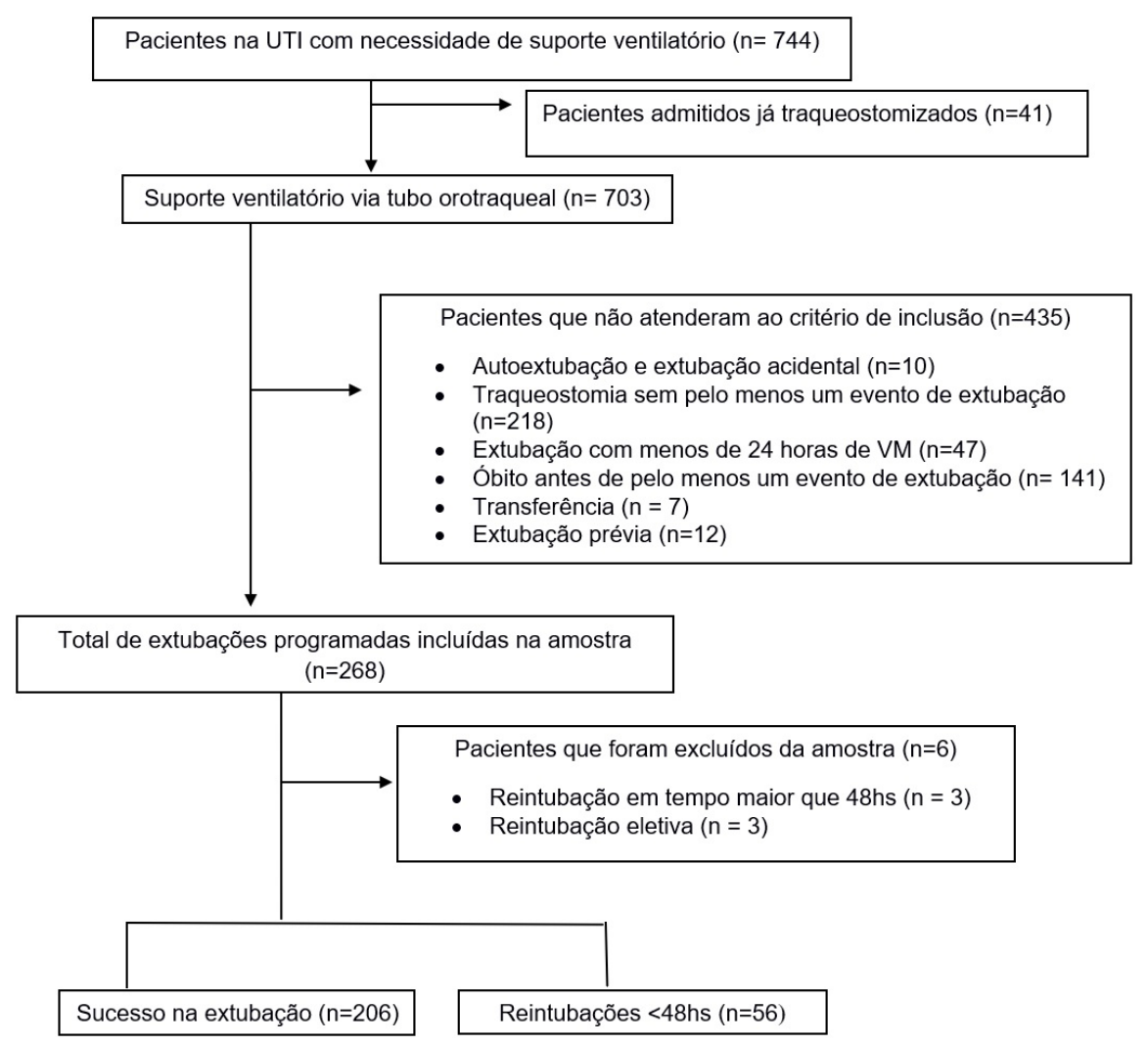

Figura 1. Fluxograma de inclusão no estudo. 
$\mathrm{O}$ teste de Mann-Whitney mostrou que a idade tem efeito no desfecho reintubação ( $U=4052,5 ; p=0,001)$. A Odds Ratio para indivíduos mais velhos serem reintubados é de 1,02 (IC95\%:1,00-1,04). A média de idade dos indivíduos reintubados foi de 50,23 $\pm 2,24$ anos (IC95\%:45,72-54,74), e dos indivíduos não reintubados, de 37,26 $\pm 2,23$ anos (IC95\%: 32,78-41,74). Além disso, os indivíduos que foram reintubados apresentaram maiores valores de APACHE II $(14,75 \pm 5,39)$ quando comparados aos não reintubados $(12,61 \pm 5,73 ; p=0,013$; IC95\%: $-3,81 ;-0,45)$ e maior tempo de VMI até a extubação $(7,50 \pm 3,41$ dias vs. 5,32 $\pm 3,31$ dias, $p=0,001$ ). Foram observados maiores valores de FR no momento da extubação para os reintubados quando comparados aos não reintubados $(22,41 \pm 5$, 49 irpm vs. 20,09 $\pm 4,93$ irpm, respectivamente; $\mathrm{p}=0,002$ ).

A causa mais frequente para intubação orotraqueal (IOT) foi cirúrgica $(35,1 \%)$ seguida por causas neurológicas $(27,8 \%)$. A regressão logística binária mostrou que pacientes que foram intubados por complicações respiratórias tiveram 4,4 mais chances de serem reintubados (OR=4,4, IC95\%:1,792-11,025, p=0,04) (Tabela 1). Igualmente, as comorbidades respiratórias pré-existentes à IOT foram preditores de reintubação. Pacientes com doenças respiratórias apresentaram 3,3 vezes mais chances de falhar à uma extubação planejada $(\mathrm{OR}=3,3$, IC95\%:1,136-9,926, p=0,05).

A causa mais comum para reintubação foi relacionada às vias aéreas (VAs) (50\%), ou seja, inabilidade de protegê-las ou presença de estridor laríngeo; seguida por alteração do padrão respiratório (37,5\%) identificado por meio da presença de esforço respiratório, alteração na FR, ou queda de saturação. A Tabela 1 a seguir apresenta estes e outros resultados.

Comparando os resultados clínicos entre os dois grupos, os seguintes desfechos foram associados com episódios de reintubação: necessidade do uso de VNI após o primeiro evento de extubação $(p<0,001)$, necessidade de traqueostomia $(p<0,001)$, e maior tempo de internação na UTI. O grupo de pacientes reintubados teve tempo de internação quase duas vezes maior $(19,27 \pm 9$, 90 dias vs. $10,47 \pm 5,38$ dias, $p<0,001)$ e também maior risco relativo de internação por um período maior que 14 dias ( $R R=0,197$, IC95\%:0,12-0,32, $p<0,001)$. Além disso, a incidência de mortalidade foi significativamente maior para os pacientes que falharam à extubação: 4,1 vezes mais chances de evoluírem para óbito ( $R R=4,1, \mathrm{IC} 95 \%: 2,87-6,19, \mathrm{p}<0,001)$.

Não foram encontradas diferenças estatisticamente significativas entre os grupos com relação às variáveis sexo $(p=0,590)$, diagnóstico clínico na admissão, tipo do TRE por pressão suporte ventilatória (PSV) vs. Tubo $T(p=0,534), P C R$ $(p=0,262)$, Hemoglobina maior ou menor que $7(p=0,507)$ ou valores absolutos $(p=0,239)$, valores gasométricos $\mathrm{pH}$ $(p=0,471), p O 2(p=0,587), p C O 2(p=0,061), \mathrm{HCO}(p=0,323)$, FC $(p=0,398)$, PAM $(p=0,536)$, febre $(p=0,612)$ e balanço hídrico 24 horas antes da extubação $(p=0,239)$, e tempo total de VMI $(p=0,070)$. A Tabela 2 a seguir apresenta a sumarização destes resultados.

Com relação aos parâmetros do checklist de extubação, pico de fluxo de tosse $(p=0,118)$, hipersecretividade $(p=0,293)$, avaliação subjetiva da tosse $(p=0,393)$ e resposta aos comandos verbais $(p=1,000)$ também não houve diferença estatisticamente significativa. Como apenas 79 dos 166 checklists foram preenchidos na íntegra (44,6\%), estes resultados podem ter sido influenciados. A Tabela 3 apresenta estes e outros resultados.

\section{DISCUSSÃO}

Este estudo identificou que idade avançada, maior escore no APACHE II, FR elevada após a extubação e intubação por causas respiratórias foram fatores importantes e preditores de reintubação em uma coorte de pacientes vítimas de trauma. Nesta amostra também houve associação entre reintubação e maior tempo de internação, maior taxa de mortalidade, necessidade de VNI pós-extubação e evolução para traqueostomia.

Os valores da taxa de reintubação nas primeiras 48 encontrados no presente estudo $(21,1 \%)$ foram maiores que os reportados por Brown et al. ${ }^{32} \mathrm{e}$ Weinberg et al. ${ }^{33}$ em pacientes de trauma (6\%) e por Lai et al. ${ }^{1}$ Lee et al. ${ }^{4}$ Nemer et al. ${ }^{10}$ e Timothy Mil et al. ${ }^{20}$ em pacientes críticos (10-15\%). Estudos realizados em outro contexto clínico e hospitalar de terapia intensiva que avaliaram pacientes com baixo risco de falha de extubação encontraram taxas menores (10-20\%); enquanto taxas mais altas (25-30\%) foram encontradas para pacientes identificados como de alto risco para insucesso ${ }^{21,34}$. Os fatores associados ao alto risco são: idade acima de 65 anos, presença de mais de uma comorbidade, doenças respiratórias ou cardíacas, escores APACHE II maior ou igual a 12, e pacientes com tosse fraca e/ou estridor laríngeo após a primeira tentativa de extubação $0^{34,35}$. Visto que o perfil de pacientes do estudo pode ser considerado de alto risco para reintubação devido à sua complexidade, incluindo traumas cranioencefálicos graves, choques hemorrágicos e sépticos, politraumas (lesões torácicas e abdominais profundas), e abstinência associado com uso recreacional de drogas e/ou álcool, a taxa de reintubação deste estudo corrobora com os achados da literatura.

Pesquisadores acreditam que taxa de reintubação muito baixa pode refletir exposição prolongada à ventilação a fim de se evitar uma possível falha; por outro lado, taxas muito elevadas podem retratar extubações precoces e manejo inadequado 15,20

Assim como encontrado no presente estudo, os pacientes com maior média de idade tiveram maior risco de serem reintubados, corroborando com outros autores, que demonstraram que a idade mais elevada é fator de risco para falha de extubação $0^{34,36,37}$. Igualmente, foi possível identificar que houve diferença da frequência respiratória entre os grupos, sendo que os reintubados 
Tabela 1. Comparação das variáveis pré extubação em relação aos indivíduos.

\begin{tabular}{|c|c|c|c|}
\hline Variáveis & Não reintubados n=206 & Reintubados $n=56$ & $p$-valor \\
\hline \multicolumn{4}{|l|}{ Sexo, n (\%) } \\
\hline Masculino & $147(77,8 \%)$ & $42(22,2 \%)$ & \multirow{2}{*}{$0,591^{1}$} \\
\hline Feminino & $59(80,8 \%)$ & $14(19,2 \%)$ & \\
\hline Idade, média \pm DP & $37,26 \pm 2,23$ & $50,23 \pm 2,24$ & $0,001^{3 *}$ \\
\hline APACHE II, média \pm DP & $12,61 \pm 5,73$ & $14,75 \pm 5,39$ & $0,013^{4 *}$ \\
\hline \multicolumn{4}{|l|}{ Causas da intubação, n (\%) } \\
\hline Vias aéreas superiores & $29(14,1 \%)$ & $5(8,9 \%)$ & $0,309^{1}$ \\
\hline Respiratórias & $21(10,2 \%)$ & $14(25,0 \%)$ & $0,004^{1 *}$ \\
\hline Instabilidade Hemodinâmica & $23(11,2 \%)$ & $5(8,9 \%)$ & $0,631^{1}$ \\
\hline Neurológica & $53(25,7 \%)$ & $20(35,7 \%)$ & $0,139^{1}$ \\
\hline Cirúrgico & $80(38,8 \%)$ & $12(21,4 \%)$ & $0,016^{1 *}$ \\
\hline \multicolumn{4}{|l|}{ Diagnóstico, n (\%) } \\
\hline Politrauma & $93(45,1 \%)$ & $18(32,1 \%)$ & $0,081^{1}$ \\
\hline Clínico & $34(16,5 \%)$ & $11(19,6 \%)$ & $0,581^{1}$ \\
\hline Trauma raquimedular & $4(1,9 \%)$ & $2(3,6 \%)$ & $0,611^{2}$ \\
\hline Trauma Crânio Encefálico & $52(25,2 \%)$ & $17(30,4 \%)$ & $0,441^{1}$ \\
\hline Intoxicação medicamentosa & $21(10,2 \%)$ & $8(14,3 \%)$ & $0,387^{1}$ \\
\hline Grande Queimado & $2(1,0 \%)$ & $0(0,0 \%)$ & $1,000^{2}$ \\
\hline \multicolumn{4}{|l|}{ Comorbidade, n (\%) } \\
\hline Cardiovascular & $37(18,0 \%)$ & $10(17,9 \%)$ & $0,986^{1}$ \\
\hline Diabetes & $15(7,3 \%)$ & $6(10,7 \%)$ & $0,409^{2}$ \\
\hline Obesidade & $3(1,5 \%)$ & $1(1,8 \%)$ & $1,000^{2}$ \\
\hline Respiratória & $9(4,4 \%)$ & $7(12,5 \%)$ & $0,051^{2 *}$ \\
\hline \multicolumn{4}{|l|}{ Teste de respiração espontânea, n(\%) } \\
\hline PSV & $195(94,7 \%)$ & $52(92,9 \%)$ & $0,534^{2}$ \\
\hline Tubo-T & $11(5,3 \%)$ & $4(7,1 \%)$ & $0,534^{2}$ \\
\hline $\begin{array}{l}\text { Tempo de VM até a extubação, média } \pm \text { DP } \\
\text { (dias) }\end{array}$ & $5,32 \pm 3,31$ & $7,50 \pm 3,41$ & $0,001^{3 *}$ \\
\hline PCR mg/dL, média \pm DP & $180,51 \pm 99,53$ & $200,92 \pm 124,31$ & $0,262^{3}$ \\
\hline Hemoglobina g/dL, média \pm DP & $9,18 \pm 1,75$ & $9,50 \pm 1,88$ & $0,239^{3}$ \\
\hline Hemoglobina Menor que 7 & $10(4,9)$ & $4(7,1)$ & \multirow{2}{*}{$0,507^{1}$} \\
\hline Hemoglobina Maior que 7 & $196(95,1)$ & $52(92,9)$ & \\
\hline $\mathrm{PaO}_{2} \mathrm{mmHg}$, média $\pm \mathrm{DP}$ & $111,47 \pm 32,68$ & $116,63 \pm 41,41$ & $0,587^{3}$ \\
\hline $\mathrm{PaCO}_{2} \mathrm{mmHg}$, média $\pm \mathrm{DP}$ & $41,48 \pm 6,77$ & $40,09 \pm 6,06$ & $0,061^{3}$ \\
\hline $\mathrm{HCO}_{3} \mathrm{mEq} / \mathrm{L}$, média $\pm \mathrm{DP}$ & $26,66 \pm 3,67$ & $26,30 \pm 4,06$ & $0,323^{3}$ \\
\hline PH arterial, média \pm DP & $7,42 \pm 0,06$ & $7,43 \pm 0,05$ & $0,471^{3}$ \\
\hline Balanço hídrico $(\mathrm{ml})$, média $\pm \mathrm{DP}$ & $522,95 \pm 1198,19$ & $598,65 \pm 1268,19$ & $0,239^{3}$ \\
\hline FR (irpm), média \pm DP & $20,09 \pm 4,93$ & $22,41 \pm 5,49$ & $0,002^{3 *}$ \\
\hline PAM mmHg, média \pm DP & $95,50 \pm 12,90$ & $94,72 \pm 13,34$ & $0,536^{3}$ \\
\hline Frequência cardíaca (bpm), média \pm DP & $100,30 \pm 16,79$ & $97,15 \pm 14,72$ & $0,398^{3}$ \\
\hline Febre $\left({ }^{\circ} \mathrm{C}\right)$ & $38(18,4)$ & $12(21,4)$ & $0,612^{1}$ \\
\hline
\end{tabular}

Legenda: APACHE II: Acute Physiology and Chronic Health Evaluation; DP: desvio padrão; PSV: pressão suporte ventilatória; VM: ventilação mecânica; PCR: proteína $\mathrm{C}$ reativa; $\mathrm{PaO}_{2}$ : pressão arterial parcial de oxigênio; $\mathrm{PaCO}_{2}$ : pressão arterial parcial de dióxido de carbono; $\mathrm{HCO}_{3}$ : bicarbonato arterial; $\mathrm{FR}$ : frequência respiratória; PAM: pressão arterial média. ${ }^{1}$ Teste de Qui-Quadrado; ${ }^{2}$ Teste exato de Fisher, ${ }^{3}$ Teste de Mann-Whitney, ${ }^{4}$ Teste t. * Significância estatística preconizada de $\mathrm{p}<0,05$. 
Tabela 2. Comparação das variáveis pós extubação em relação aos indivíduos não reintubados e reintubados.

\begin{tabular}{|c|c|c|c|}
\hline Variáveis & Não reintubados $n=206$ & Reintubados $n=56$ & $p$-valor \\
\hline VNI após a extubação, n (\%) & $14(6,8 \%)$ & $21(37,5 \%)$ & $<0,001^{2 *}$ \\
\hline Traqueostomia, n (\%) & $1(0,5 \%)$ & $37(66,1 \%)$ & $<0,001^{2 *}$ \\
\hline Tempo total em VM, média \pm DP (dias) & $5,34 \pm 3,14$ & $13,86 \pm 6,33$ & $<0,070^{3}$ \\
\hline Tempo total de internação na UTI, média \pm DP (dias) & $10,47 \pm 5,38$ & $19,27 \pm 9,90$ & $<0,001^{3 *}$ \\
\hline \multicolumn{4}{|l|}{ Tempo de internação na UTI, n (\%) } \\
\hline$\leq 14$ dias & $167(81,1 \%)$ & $18(32,1 \%)$ & \multirow{2}{*}{$<0,001^{1 *}$} \\
\hline$>14$ dias & $39(18,9 \%)$ & $38(67,9 \%)$ & \\
\hline Mortalidade na UTI & $6(2,9 \%)$ & $15(26,8 \%)$ & $<0,001^{2 *}$ \\
\hline
\end{tabular}

Legenda: VNI: ventilação não invasiva; DP: desvio padrão; VM: ventilação mecânica; UTI: Unidade de Terapia Intensiva. ${ }^{1}$ Teste de Qui-Quadrado, ${ }^{2}$ Teste exato de Fisher, ${ }^{3}$ Teste de Mann-Whitney. *Significância estatística preconizada de $\mathrm{p}<0,05$.

Tabela 3. Check List de Extubação.

\begin{tabular}{lccc}
\hline \multicolumn{1}{c}{ Check List $\mathbf{n}(\%)$} & Não reintubados $\mathbf{n = 6 3}$ & Reintubados n=16 & $p$-valor \\
\hline Peak Flow $<\mathbf{6 0}$ (L/min) & $3(4,8 \%)$ & $3(18,8 \%)$ & $0,094^{2}$ \\
Avaliação Subjetiva da Tosse $<\mathbf{2}$ & $1(1,6 \%)$ & $1(6,3 \%)$ & $0,366^{2}$ \\
Não responde a pelo menos 2 Comandos Verbais & $3(4,8 \%)$ & $0(0,0 \%)$ & $1,000^{2}$ \\
Apresenta Hipersecretividade & $8(12,7 \%)$ & $0(0,0 \%)$ & $0,197^{2}$ \\
\hline
\end{tabular}

Legenda: ${ }^{1}$ Teste de Qui-Quadrado, ${ }^{2}$ Teste exato de Fisher, ${ }^{3}$ Teste de Mann-Whitney; *Significância estatística preconizada de $\mathrm{p}<0,05$.

apresentaram maiores valores no momento da extubação. Além disso, assim como os achados de Wen-Lin Su et al. ${ }^{37}$ e Mohammad Khamiees et al. ${ }^{18}$, diferenças significativas entre as pontuações do APACHE II foram encontradas entre os pacientes que tiveram sucesso na extubação e os que falharam, sendo maiores para o último grupo. Uma possível explicação para estes resultados é o fato de que os pacientes do presente estudo são de alta complexidade e apresentam doenças graves.

Este estudo demonstrou que o tempo de VMI maior que sete dias está relacionado a maiores chances de falha na extubação. O resultado é consistente com outros estudos que demonstraram que uso de VMI por um período maior que 7 dias aumentou entre 4 e 7 vezes a chance de reintubação 2,4,12,21. Ademais, assim como demonstrado previamente, episódios de reintubação podem piorar diretamente os desfechos de pacientes, independente da gravidade da doença subjacente ${ }^{17,20,21,35,36,38}$. Neste estudo, pacientes que exigiram reintubação apresentaram 3,3 vezes maior tempo de internação na UTI e aumento da mortalidade em aproximadamente 4 vezes. Maior mortalidade desses pacientes pode ser atribuída ao possível desenvolvimento de nova condição clínica durante o intervalo entre extubação e reintubação ou até mesmo após a reintubação.

Outro fator de risco associado à reintubação foi o motivo que levou à IOT. Assim como encontrado por Silva-Cruz², pacientes intubados por causas respiratórias tiveram aproximadamente 4 vezes mais chances de falhar à extubação. Igualmente, foi possível identificar que a maioria dos pacientes que não obtiveram sucesso na extubação apresentavam comorbidades respiratórias, corroborando com os achados de Thile ${ }^{12}$ e Atul e Vandana ${ }^{39}$.

Assim como nos achados de Lee et al. ${ }^{4}$, Frutos-Vivar et al. ${ }^{35}$ e Miltiades et al. ${ }^{36}$ o uso de VNI pós-extubação foi associado com maior chance de falência à extubação, e seu uso foi significativamente mais elevado em pacientes que falharam à extubação. A análise deste dado deve ser feita com cuidado, já que a VNI pode ter sido indicada para os pacientes de alto risco para falência ou como tentativa de se evitar ou até mesmo postergar a reintubação.

Entre os motivos para reintubação, causas relacionadas às VAs foram mais comuns seguida por alterações do padrão respiratório. Assim como encontrado em outros estudos, a inabilidade de proteção das VAs e/ou a presença de estridor laríngeo estão associados com maior chance de reintubação 7,12,18,19,37,39,40. Outro desfecho clínico que demonstrou relação estatisticamente significativa com os episódios de reintubação foi a necessidade de traqueostomia. Um resultado similar ao encontrado por Kulkarni e Agarwa| ${ }^{39}$ e Seymour et al..$^{41}$ que verificaram associação entre episódios de reintubação com necessidade de manutenção de via aérea artificial por meio da traqueostomia. 
Ao contrário do que muitos estudos mostram, as medidas da força de tosse (avaliação subjetiva e por meio do Peak Flow meter), hipersecretividade e resposta a comandos verbais não apresentaram associação em conjunto nem individualmente com os episódios de reintubação8 $818,20,21,23,37$. A subjetividade envolvida com a realização de algumas avaliações e a variedade de profissionais envolvidos na realização das medidas pode ter contribuído para uma inconsistência na aplicação das medidas e na classificação das mesmas.

Assim como descrito por Thille et al. ${ }^{34}$ e FrutosVivar et al. ${ }^{35}$, foi encontrada correlação entre o surgimento de pneumonia associada à VMI (PAV) com reintubação. No presente estudo, os pacientes que tiveram diagnóstico de PAV após o episódio de extubação tiveram 34 vezes mais chances de serem reintubados, sugerindo que pacientes possam ter sido extubados com um processo infeccioso já em evolução, o que pode ter contribuído para o desfecho. Igualmente, pacientes que foram diagnosticados com sepse após à extubação, tiveram aproximadamente 14 vezes mais chances de retornarem à VMI. Entretanto, como o intervalo de tempo (dias) de diagnóstico de PAV ou sepse foi variado entre os pacientes reintubados, pode ser que esses eventos tenham sido decorrentes do segundo momento de uso de VMI, ou seja, após a reintubação e não tenham tido relação com a falência após a primeira tentativa de extubação. Portanto, esses dados devem ser analisados com cautela, uma vez que o intervalo de confiança se mostrou bastante amplo.

Este estudo apresentou algumas limitações. Primeiramente, o protocolo de extubação (checklist) foi elaborado e implementado durante o período do estudo, deixando alguns pacientes sem avaliação dos parâmetros estabelecidos. Portanto, extubações precoces ou tardias podem ter acontecido sem que todas as variáveis fossem coletadas. Além disso, a equipe teve pouco tempo para familiarização com a execução e avaliação das medidas, podendo acarretar em inconsistência na obtenção dos dados. Finalmente, alguns fatores descritos na literatura não puderam ser avaliados devido à perda de dados e/ou dados incompletos.

Apesar dessas limitações no presente estudo, os episódios de reintubação estiveram diretamente relacionados à incapacidade de proteger VAs e a presença estridor laríngeo, portanto, é recomendado o uso de medidas quantitativas bem descritas na literatura que possam favorecer a adequada avaliação de permeabilidade e proteção das VAs. Protocolos de extubação talvez não possam ser generalizados para diferentes perfis de pacientes, sendo assim, estudos práticos com intuito de avaliar e validar os mesmos podem reduzir a incidência de falha de extubação nesse perfil de população.

\section{CONCLUSÃO}

Conclui-se que a falha de extubação dentro de um período de 48 horas foi associada a um maior tempo de internação, maior mortalidade, necessidade de VNI pósextubação e evolução para traqueostomia. Além disso, foram fatores de risco independentes para reintubação a idade avançada, maior escore no APACHE II, FR elevada pós-extubação, IOT por causas respiratórias e a presença de comorbidades respiratórias.

\section{FONTE DE FINANCIAMENTO}

Nada a declarar.

\section{CONFLITO DE INTERESSE}

Nada a declarar.

\section{REFERÊNCIAS}

1. Lai CC, Chen CM, Chiang SR, Liu WL, Weng SF, Sung MI, et al. Establishing predictors for successfully planned endotracheal extubation. Medicine (United States). 2016;95(41):e4852. http://dx.doi.org/10.1097/MD.0000000000004852. PMid:27741103.

2. Silva-Cruz AL, Velarde-Jacay K, Carreazo NY, EscalanteKanashiro R. Risk factors for extubation failure in the intensive care unit. Rev Bras Ter Intensiva. 2018;30(3):294300. http://dx.doi.org/10.5935/0103-507X.20180046. PMid:30304083.

3. Tanios M, Epstein S, Grzeskowiak M, Nguyen HM, Park $\mathrm{H}$, Leo J. Influence of sedation strategies on unplanned extubation in a mixed intensive care unit. Am J Crit Care. 2014;23(4):306-14. http://dx.doi.org/10.4037/ajcc20144446. PMid:24986171.

4. Lee ESH, Lim DTJ, Taculod JM, Sahagun JT, Otero JP, Teo $\mathrm{K}$, et al. Factors associated with reintubation in an intensive care unit: A prospective observational study. Indian J Crit Care Med. 2017;21(3):131-7. http://dx.doi.org/10.4103/ijccm. IJCCM_452_16. PMid:28400683.

5. Nemer SN, Barbas CSV. Índices de desmame: o que devemos saber? Weaning indexes: what do we need to know? Pulmão RJ. 2011;20(3):24-8.

6. Khamiees M, Raju P, DeGirolamo A, Amoateng-Adjepong Y, Manthous CA. Predictors of extubation outcome in patients who have successfully completed a spontaneous breathing trial. Chest. 2001;120(4):1262-70. http://dx.doi.org/10.1378/ chest.120.4.1262. PMid:11591570.

7. Hyzy R. Extubation management in the adult intensive care. Waltham, MA: UpToDate Inc.; 2020.

8. Salam A, Tilluckdharry L, Amoateng-Adjepong Y, Manthous CA. Neurologic status, cough, secretions and extubation outcomes. Intensive Care Med. 2004;30(7):1334-9. http:// dx.doi.org/10.1007/s00134-004-2231-7. PMid:14999444.

9. Lee ESH, Lim DTJ, Taculod JM, Sahagun JT, Otero JP, Teo $\mathrm{K}$, et al. Factors Associated with Reintubation in an Intensive Care Unit: A Prospective Observational Study. Indian J Crit Care Med. 2017;21(3):131-7. http://dx.doi.org/10.4103/ijccm. IJCCM_452_16. PMid:28400683.

10. Nemer SN, Barbas CSV. Parâmetros preditivos para o desmame da ventilação mecânica. J Bras Pneumol. 
2011;37(5):669-79. http://dx.doi.org/10.1590/S180637132011000500016. PMid:22042401.

11. Barbas CSV, Isola AM, Farias AMC. Diretrizes Brasileiras de Ventilação Mecânica. Brasília: Associação de Medicina Intensiva Brasileira; 2013.

12. Thille AW, Boissier F, Ben Ghezala H, Razazi K, MekontsoDessap A, Brun-Buisson C. Risk factors for and prediction by caregivers of extubation failure in ICU patients: A prospective study. Crit Care Med. 2015;43(3):613-20. http://dx.doi. org/10.1097/CCM.0000000000000748. PMid:25479115.

13. Thille AW, Richard JCM, Brochard L. The decision to extubate in the intensive care unit. Am J Respir Crit Care Med. 2013;187(12):1294-302. http://dx.doi.org/10.1164/ rccm.201208-1523Cl. PMid:23641924.

14. Weinberg JA, Stevens LR, Goslar PW, Thompson TM, Sanford JL, Petersen SR. Risk factors for extubation failure at a level i trauma center: does the specialty of the intensivist matter? Trauma Surgery and Acute Care Open. 2016;1(1):e000052. http://dx.doi.org/10.1136/ tsaco-2016-000052. PMid:29766074.

15. Artime CA, Hagberg CA. Tracheal extubation. Respir Care. 2014;59(6):991-1005. http://dx.doi.org/10.4187/ respcare.02926. PMid:24891203.

16. Maggiore SM, Battilana M, Serano L, Petrini F. Ventilatory support after extubation in critically ill patients. Lancet Respir Med. 2018;6(12):948-62. http://dx.doi.org/10.1016/ S2213-2600(18)30375-8. PMid:30629933.

17. Vetrugno L, Guadagnin GM, Brussa A, Orso D, Garofalo E, Bruni A, et al. Mechanical ventilation weaning issues can be counted on the fingers of just one hand: part 1. Ultrasound Journal. 2020;12(1):9. http://dx.doi.org/10.1186/s13089-02000161-y. PMid:32166566.

18. Khamiees M, Raju P, DeGirolamo A, Amoateng-Adjepong Y, Manthous CA. Predictors of extubation outcome in patients who have successfully completed a spontaneous breathing trial. Chest. 2001;120(4):1262-70. http://dx.doi.org/10.1378/ chest.120.4.1262. PMid:11591570.

19. Smailes ST, McVicar AJ, Martin R. Cough strength, secretions and extubation outcome in burn patients who have passed a spontaneous breathing trial. Burns. 2013;39(2):23642. http://dx.doi.org/10.1016/j.burns.2012.09.028. PMid:23107354.

20. Miu T, Joffe AM, Yanez ND, Khandelwal N, Dagal AH, Deem $\mathrm{S}$, et al. Predictors of reintubation in critically III patients. Respir Care. 2014;59(2):178-85. http://dx.doi.org/10.4187/ respcare.02527. PMid:23882103.

21. Thille AW, Cortés-Puch I, Esteban A. Weaning from the ventilator and extubation in ICU. Curr Opin Crit Care. 2013;19(1):57-64. http://dx.doi.org/10.1097/ MCC.0b013e32835c5095. PMid:23235542.

22. Li Y, Li H, Zhang D. Comparison of T-piece and pressure support ventilation as spontaneous breathing trials in critically ill patients : a systematic review and meta-analysis. Crit Care. 2020;24:1-10.

23. Smina M, Salam A, Khamiees M, Gada P, AmoatengAdjepong Y, Manthous CA. Cough peak flows and extubation outcomes. Chest. 2003;124(1):262-8. http://dx.doi. org/10.1378/chest.124.1.262. PMid:12853532.

24. Goldwasser R, Farias A, Freitas EE, Saddy F, Amado V, Okamoto V. Desmame e interrupção da ventilação mecânica. J Bras Pneumol. 2007;33(Suppl. 2):384-92. http://dx.doi.org/10.1590/S1806-37132007000800008. PMid:18026671.

25. Jung B, Vaschetto $R$, Jaber $S$. Ten tips to optimize weaning and extubation success in the critically ill. Intensive Care
Med. 2020;46(12):2461-3. http://dx.doi.org/10.1007/s00134020-06300-2. PMid:33104823.

26. Torres A, Gatell JM, Aznar E, el-Ebiary M, Puig de la Bellacasa J, GonzálezJ, et al. Re-intubation increases the risk of nosocomial pneumonia in patients needing mechanical ventilation. Am J Respir Crit Care Med. 1995;152(1):137-41. http://dx.doi. org/10.1164/ajrccm.152.1.7599812. PMid:7599812.

27. Bonten MJM, Kollef MH, Hall JB. Risk factors for ventilatorassociated pneumonia: from epidemiology to patient management. Clin Infect Dis. 2004;38(8):1141-9. http:// dx.doi.org/10.1086/383039. PMid:15095221.

28. Jiang C, Esquinas A, Mina B. Evaluation of cough peak expiratory flow as a predictor of successful mechanical ventilation discontinuation: A narrative review of the literature. J Intensive Care. 2017;5(1):1-5. http://dx.doi. org/10.1186/s40560-017-0229-9. PMid:28588895.

29. Bai L, Duan J. Use of cough peak flow measured by a ventilator to predict re-intubation when a spirometer is unavailable. Respir Care. 2017;62(5):566-71. http://dx.doi. org/10.4187/respcare.05260. PMid:28246308.

30. Gobert F, Yonis H, Tapponnier R, Fernandez R, Labaune $\mathrm{MA}$, Burle JF, et al. Predicting extubation outcome by cough peak flow measured using a built-in ventilator flow meter. Respir Care. 2017;62(12):1505-19. http://dx.doi.org/10.4187/ respcare.05460. PMid:28900041.

31. Duan BJ, Zhou L, Xiao M, Liu J, Yang X. Semiquantitative cough strength score for predicting reintubation after planned extubation. Pulmonary Critical Care. 2015;24(6):86-91.

32. Brown CVR, Daigle JB, Foulkrod KH, Brouillette B, Clark A, Czysz C, et al. Risk factors associated with early reintubation in trauma patients: A prospective observational study. J Trauma Inj Infect Crit Care. 2011;71(1):37-41, discussion 41-2. http://dx.doi.org/10.1097/TA.0b013e31821e0c6e. PMid:21818012.

33. Weinberg JA, Stevens LR, Goslar PW, Thompson TM, Sanford $J L$, Petersen SR. Risk factors for extubation failure at a level i trauma center: does the specialty of the intensivist matter? Trauma Surgery and Acute Care Open. 2016;1(1):1-5. http:// dx.doi.org/10.1136/tsaco-2016-000052. PMid:29766074.

34. Thille AW, Harrois A, Schortgen F, Brun-Buisson C, Brochard L. Outcomes of extubation failure in medical intensive care unit patients. Crit Care Med. 2011;39(12):2612-8. http://dx.doi.org/10.1097/CCM.0b013e3182282a5a. PMid:21765357.

35. Frutos-Vivar F, Esteban A, Apezteguia C, González M, Arabi $Y$, Restrepo $\mathrm{Ml}$, et al. Outcome of reintubated patients after scheduled extubation. J Crit Care. 2011;26(5):502-9. http:// dx.doi.org/10.1016/j.jcrc.2010.12.015. PMid:21376523.

36. Miltiades AN, Gershengorn HB, Hua M, Kramer AA, Li G, Wunsch $\mathrm{H}$. Cumulative probability and time to reintubation in U.S. ICUs. Crit Care Med. 2017;45(5):835-42. http://dx.doi. org/10.1097/CCM.0000000000002327. PMid:28288027.

37. Su WL, Chen YH, Chen CW, Yang SH, Su CL, Perng WC, et al. Involuntary cough strength and extubation outcomes for patients in an ICU. Chest. 2010;137(4):777-82. http://dx.doi. org/10.1378/chest.07-2808. PMid:20097804.

38. Alomía D, Coral M, Ortegón S, Soto R, Muñoz V. Risk factors associated to extubation failure in adult patients in an Intensive Care Unit of the City of Cali. Ciencias de la Salud. 2017;15(2):237. ttp://dx.doi.org/10.12804/revistas.urosario. edu.co/revsalud/a.5760

39. Kulkarni A, Agarwal V. Extubation failure in intensive care unit: predictors and management. Indian J Crit Care Med. 2008;12(1):1-9. http://dx.doi.org/10.4103/0972-5229.40942. PMid:19826583. 
40. Krivinskas S, Sarkar R, Turner O, Goonetilleke K, Anderson P. PREDICTING EXTUBATION FAILURE. Intensive Care Med Exp. 2015;3(Suppl. 1):A996. http://dx.doi.org/10.1186/2197425X-3-S1-A996.
41. Seymour CW, Martinez A, Christie JD, Fuchs BD. The outcome of extubation failure in a community hospital intensive care unit: A cohort study. Crit Care. 2004;8(5):R322-7. http:// dx.doi.org/10.1186/cc2913. PMid:15469575. 